\title{
Das Reviewboard nimmt die Arbeit auf
}

Das Jahr 2010 ist schon im Januar mit einer HeilberufeSCIENCE-Sonderausgabe zum Pflegekongress in Berlin gestartet. Aufgrund des hohen Interesses an den zwei bisher publizierten Sonderausgaben zu den Heilberufe-Kongressen sollen diese zukünftig eine fest etablierte Ergänzung zu den HeilberufeSCIENCE-Ausgaben bilden, deren erste von vier regulären Ausgaben 2010 sich jetzt im World Wide Web befindet.

HeilberufeSCIENCE entwickelt sich weiter: Ganz neu ist unser "Reviewboard", das dem Zweck einer noch besseren Begutachtung eingehender Beiträge dient und von nun an den Beirat unterstützen wird. Dazu wurden ausgewiesene Expertlnnen gewonnen, deren fachliche Kompetenz eine adäquate Auswahl an künftigen Beiträgen für HeilberufeSCIENCE sicherstellen soll. Um sich neuen wissenschaftlichen Impulsen nicht zu verschließen, erfolgt die Aufnahme in das Reviewboard nur für drei Jahre, wobei eine Verlängerung der Mitgliedschaft um jeweils weitere drei Jahre möglich ist. Mit Hilfe der jeweiligen Reviewer kann nun noch intensiver mit den AutorInnen der übersandten Manuskripte im kollegialen Austausch über die endgültige Gestaltung der Beiträge diskutiert werden. Da HeilberufeSCIENCE den wissenschaftlichen Nachwuchs in den Disziplinen Pflegemanagement, Pflegepädagogik, Pflegewissenschaften, Gerontologie, Altenpflege und Gesundheitswissenschaften/Public Health fördern möchte, soll dieser fachliche Austausch insbesondere jüngeren AutorInnen bei der Erstellung von Beiträgen helfen. Beiträge von Nachwuchswissenschaftlerlnnen aus dieser Zielgruppe sind also weiterhin sehr willkommen.

In der akutellen Ausgabe werden Ihnen fünf Artikel zu unterschiedlichen Themen geboten: Der Beitrag von Romeyke und Stummer setzt sich mit der Kosteneffizienz und Qualitätssicherung durch klinische Behandlungspfade in der stationären Versorgung von Patienten auseinander. Die Beiträge von Fleischer und Rester et al. bieten Untersuchungen aus dem Gebiet der stationären Pflege. Dabei setzt sich der Artikel von Rester et al. anhand einer Literaturübersicht mit gruppenbezogenen Wohnformen in der Langzeitpflege auseinander. Die Untersuchung von Fleischer geht in einer methodisch orientierten Arbeit auf die psychischen Belastungen und Beanspruchungen von Pflegepersonal in einer stationären Altenpflegeeinrichtung ein und zeigt, wie diese einfach und praktikabel ermittelt werden können. Einen weiteren methodisch orientierten Beitrag liefern Müller et al., die die Interrater-Reliabilität der deutschen Autar-DVT (Deep Vein Thrombosis)-Skala zur Einschätzung des Thromboserisikos untersucht haben. Den Abschluss bildet die Arbeit von

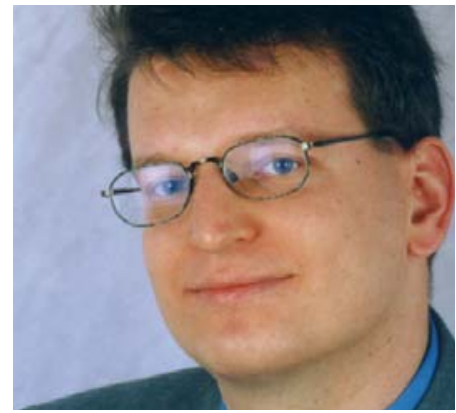

Prof. Dr. med. habil. Jörg Klewer, Zwickau

Schriftleitung

heilberufescience@springer.com

HeilberufeSCIENCE 2010; 1 (1): 1 DOI 10.1007/s16024-010-0125-x

Grünbeck, die das Gesundheitsverhalten von Studierenden in den Bachelor-Studiengängen Pflegemanagement und Gesundheitsmanagement unter die Lupe genommen hat und Ansätze zur Gesundheitsförderung bei diesen zukünftigen Führungskräften im Gesundheitssystem aufzeigt.

Nun möchte ich Sie wieder einladen, diese fünf Beiträge kritisch zu lesen und in den wissenschaftlichen Dialog einzutreten und natürlich würde es mich freuen, Sie in der nächsten Ausgabe von HeilberufeSCIENCE im Mai wieder an dieser Stelle begrüßen zu können.

Ihr

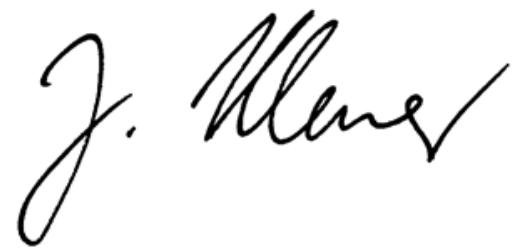

